\title{
EFFECTS OF SURFACE ROUGHNESS ON POROUS INCLINED SLIDER BEARINGS LUBRICATED WITH MICROPOLAR FLUIDS
}

\author{
N.B. Naduvinamani \\ Department of Mathematics, Gulbarga University, Gulbarga-585 106, INDIA., naduvinamaninb@yahoo.co.in \\ T.V. Biradar \\ Department of Mathematics, Appa Institute of Engineering and Technology, Gulbarga-585 103, INDIA.
}

Follow this and additional works at: https://jmstt.ntou.edu.tw/journal

Part of the Engineering Commons

\section{Recommended Citation}

Naduvinamani, N.B. and Biradar, T.V. (2007) "EFFECTS OF SURFACE ROUGHNESS ON POROUS INCLINED SLIDER BEARINGS LUBRICATED WITH MICROPOLAR FLUIDS," Journal of Marine Science and Technology. Vol. 15: Iss. 4, Article 2.

DOI: $10.51400 / 2709-6998.2044$

Available at: https://jmstt.ntou.edu.tw/journal/vol15/iss4/2

This Research Article is brought to you for free and open access by Journal of Marine Science and Technology. It has been accepted for inclusion in Journal of Marine Science and Technology by an authorized editor of Journal of Marine Science and Technology. 


\section{EFFECTS OF SURFACE ROUGHNESS ON POROUS INCLINED SLIDER BEARINGS LUBRICATED WITH MICROPOLAR FLUIDS}

\section{Acknowledgements}

The authors are grateful to the referees for their valuable comments on the earlier draft of the paper. One of the authors (TVB) is thankful to Poojya Dr. Sharnbasawappa Appa, President, Sharnbasveshwar Vidya Vardhak Sangha, Gulbarga for his encouragements. This work is supported by the UGC New Delhi under the Project No.F-31-84/2005(SR). 


\title{
EFFECTS OF SURFACE ROUGHNESS ON POROUS INCLINED SLIDER BEARINGS LUBRICATED WITH MICROPOLAR FLUIDS
}

\author{
N.B. Naduvinamani* and T.V. Biradar**
}

Key words: surface roughness, micropolar fluids, porous, slider bearings.

\begin{abstract}
This paper describes a theoretical analysis of the effect of surface roughness on the hydrodynamic lubrication of one-dimensional porous inclined slider bearings with micropolar fluids. To account for more realistic situations of the non-uniform rubbing of the bearing surfaces, it is assumed that, the probability density function for the random variable characterizing the surface roughness is asymmetrical with non-zero mean. The averaged modified Reynolds type equation is derived and the closed form expressions for the bearing characteristics are obtained. The numerical computations of the results show that, the performance of the porous inclined slider bearing is improved for the micorpolar lubricants as compared to the corresponding Newtonian lubricants. Further, it is found that, the negatively skewed surface roughness improves the porous bearing performance whereas, the bearing performance suffers due to the presence of positively skewed surface roughness.
\end{abstract}

\section{INTRODUCTION}

The study of the effects of surface roughness on the hydrodynamic lubrication of various bearing systems has been a subject of growing interest. This is mainly because of the reason that, in practice all bearing surfaces are rough. The study of the effect of surface roughness has a greater importance in the study of porous bearings as the surface roughness is inherent to the process used in their manufacture. In general, the roughness asperity height is of the same order as the mean separation between the lubricated contacts. In such situations, surface roughness affects the performance of the bearing system. The stochastic study of Tzeng and Saibel [21] has fascinated several investigators in the field of tribology. Patir and Cheng $[15,16]$ proposed an average flow model for deriving the

Paper Submitted 08/04/06, Accepted 12/14/06. Author for Correspondence: N.B. Naduvinamani. E-mail: naduvinamaninb@yahoo.co.in.

*Department of Mathematics, Gulbarga University, Gulbarga-585 106, INDIA.

**Department of Mathematics, Appa Institute of Engineering and Technology, Gulbarga-585 103, INDIA.
Reynolds type equation which is applicable to any general surface roughness structure. Chritensen [3] proposed a new stochastic averaging approach for the study of roughness effects on the hydrodynamic lubrication of bearings. Christensen and Tonder [4-6] presented a comprehensive general analysis for the two types of one dimensional surface roughness patterns Viz. transverse and longitudinal, based on the general probability density function and this approach formed the basis for the study of surface roughness effects by several researchers $[9,11,12,14]$. In all these studies it is assumed that, the probability density function for the random variable characterizing the surface roughness is symmetric with zero mean. However, in general due to non-uniform rubbing of the surfaces, especially in slider bearings the distribution of surface roughness may be asymmetrical. In view of this, Andharia et al. [2] studied the effect of surface roughness on the performance characteristics of one-dimensional slider bearings with an assumption of the probability density function for the random variable characterizing the surface roughness is asymmetrical with a non-zero mean. All these studies are limited to the study of surface roughness effects on bearing performance with Newtonian lubricants.

Most of the modern lubricants are no longer Newtonian fluids, since the use of the lubricant additives in lubricants has become a common practice in order to improve the performance of lubricants. Therefore, several microcontinuum theories [7] have been proposed to account for the effects of additives. Eringen [8] micropolar fluid theory is a subclass of microfluids that ignores the deformation of the microelements and allows for the particle micromotion to take place. Allen and Kline [1] presented the lubrication theory for micropolar fluids in which the order-ofmagnitude arguments are used to simplify the governing equations to a system of coupled, linear, ordinary differential equations. The lubrication theory for micropolar fluids and its application to a journal bearing is presented by Prakash and Sinha [17]. Shukla and Isa [20] derived a generalized Reynolds equation for 
micropolar lubricants and its application to optimum one-dimensional slider bearings. Ramanaiah and Dubey [19] presented the analysis for optimum slider profile of a slider bearing lubricated with a micropolar fluid. The micropolarity-roughness interaction in hydrodynamic lubrication is studied by Praksh et al. [18]. This is based on the Chritensen's [3] stochastic model for hydrodynamic lubrication of rough surfaces. The porous inclined slider bearing lubricated with micropolar fluid is studied by Verma et al. [22] with an assumption of perfectly smooth bearing surfaces. The effect of threedimensional irregularities on the hydrodynamic lubrication of journal bearings lubricated with micropolar lubricants is studied by Lin [13].

In this paper an attempt has been made to study the effect of micropolarity-roughness interaction on the performance of one-dimensional porous inclined slider bearings. It is assumed that, the probability density function for the random variable characterizing the surface roughness is asymmetrical with non-zero mean.

\section{FORMULATION OF THE PROBLEM}

Figure 1 shows the schematic diagram of the porous inclined slider bearing. It consists of two surfaces separated by a lubricant film. The lower surface of the porous bearing is at rest and the upper solid surface is moving in its own plane with a constant velocity U. It is assumed that the bearing surfaces are rough and infinitely wide in the z-direction. The lubricant in the film region as well as in the porous region is assumed to be micropolar fluid.

To mathematically model the surface roughness, expression for the film thickness is considered to be consisting of two parts.

$$
H=h(x)+h_{s}
$$

where

$$
h(x)=h_{1}-\left(\frac{h_{1}-h_{0}}{L}\right) x
$$

is the mean film thickness, $h_{s}$ is a randomly varying quantity measured from the mean level and thus characterizes the surface roughness and $\mathrm{L}$ is the length of the bearing. The stochastic part $h_{s}$ is assumed to have the probability density function $\mathrm{f}\left(h_{s}\right)$ defined over the domain $-\mathrm{C} \leq h_{s} \leq \mathrm{C}$ where $\mathrm{C}$ is the maximum deviation from the mean level. For a continuous non-broken fluid film thickness it is assumed that $\mathrm{C}<<\mathrm{h}(\mathrm{x})$.

The mean $\alpha^{*}$, the standard deviation $\sigma^{*}$ and the parameter $\varepsilon^{*}$, which is the measure of symmetry of the

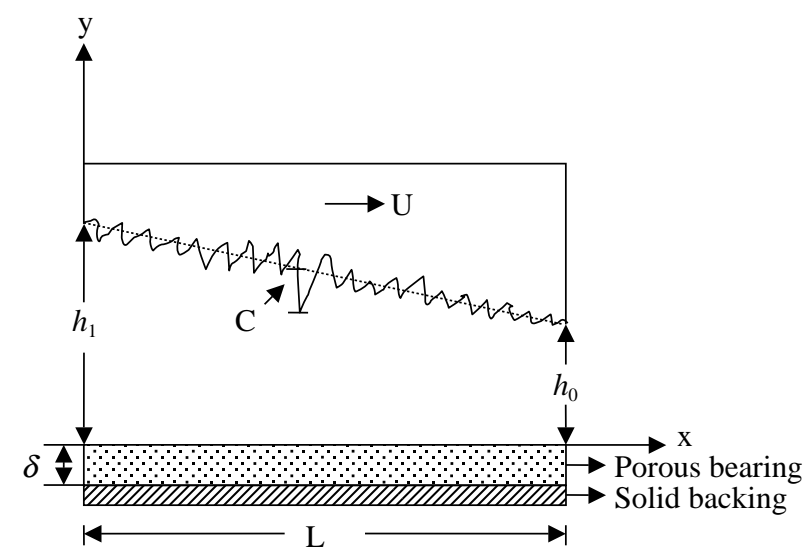

Fig. 1. Porous plane inclined slider bearing.

random variable $h_{s}$ are defined as

$$
\begin{aligned}
& \alpha^{*}=E\left(h_{s}\right) \\
& \sigma^{*}=E\left[\left(h_{s}-\alpha^{*}\right)^{2}\right] \\
& \varepsilon^{*}=E\left[\left(h_{s}-\alpha^{*}\right)^{3}\right]
\end{aligned}
$$

where $E$ is the expectation operator defined by

$$
E(\bullet)=\int_{-\infty}^{\infty}(\bullet) f\left(h_{s}\right) d h_{s}
$$

It is to be noted that, the parameters $\alpha^{*}, \sigma^{*}$ and $\varepsilon^{*}$ are independent of $\mathrm{x}$, the mean $\alpha^{*}$ and the parameter $\varepsilon^{*}$ can assume both positive and negative values however $\sigma^{*}$ can always assume positive values.

The field equations for micropolar fluids proposed by Eringen [8] simplify considerably under the usual assumptions of hydrodynamic lubrication i.e. laminar, incompressible fluid with negligible body forces and negligible inertia forces. The resulting equations under steady state conditions given by [22] are;

Conservation of mass:

$$
\frac{\partial u}{\partial x}+\frac{\partial v}{\partial y}=0
$$

Conservation of momentum:

$$
\begin{aligned}
& (\mu+K) \frac{\partial^{2} u}{\partial y^{2}}+K \frac{\partial v}{\partial y}-\frac{\partial p}{\partial x}=0 \\
& \frac{\partial p}{\partial y}=0
\end{aligned}
$$

Conservation of angular momentum: 


$$
\gamma \frac{\partial^{2} v}{\partial y^{2}}-K \frac{\partial u}{\partial y}-2 K v=0
$$

where $\gamma$ and $\mathrm{K}$ are the viscosity coefficients for micropolar fluids.

The flow of micropolar lubricants in the porous matrix is governed by the modified Darcy's law

$$
\boldsymbol{q}=\frac{-k}{\mu+K} \nabla p^{*}
$$

where $\boldsymbol{q}=\left(u^{*}, v^{*}\right)$ is the modified Darcy velocity vector, $k$ is the permeability of the porous matrix and $p^{*}$ is the pressure in the porous matrix.

The modified Reynolds equation for smooth porous inclined slider bearing lubricated with micropolar fluid was obtained by [22] in the form

$$
\frac{\partial}{\partial x}\left\{\left[f(H, M)+\frac{k \delta}{\mu+K}\right] \frac{\partial p}{\partial x}\right\}=\frac{U}{2} \frac{\partial H}{\partial x}
$$

where

$f(H, M)=\frac{h_{0}^{3}}{\mu(2+\lambda)}\left[\frac{-3 \lambda \bar{H}^{2} M+3 \lambda \bar{H} \tanh (M \bar{H})+2 \bar{H}^{3} M^{2}(1+\lambda) \tanh (M \bar{H})}{12 M^{2}(1+\lambda) \tanh (M \bar{H})}\right]$

where

$\lambda=\frac{\mu}{K}, \bar{H}=\frac{H}{h_{0}}, M=\frac{m h_{0}}{2}, m=\left[\frac{K(2 \mu+K)}{\gamma(\mu+K)}\right]^{1 / 2}$ and $\delta$ is the porous layer thickness.

Multiplying both sides of Eq.(11) by $h_{s}$ and integrating with respect to $h_{s}$ over the interval $-\mathrm{C}$ to $\mathrm{C}$ and using Eqs. (2)-(4), gives the averaged Reynolds type equation in the form

$$
\frac{\partial}{\partial x}\left\{\left[G\left(h, M, \alpha^{*}, \sigma^{*}, \varepsilon^{*}\right)+\frac{k \delta}{\mu+K}\right] \frac{\partial \bar{p}}{\partial x}\right\}=\frac{U}{2} \frac{\partial h}{\partial x}
$$

where $\bar{p}$ is the expected value of $\mathrm{p}$ and

$G\left(h, M, \alpha^{*}, \sigma^{*}, \varepsilon^{*}\right)=\frac{h_{0}^{3}}{\mu(2+\lambda)}\left[3 \lambda\left\{\frac{h}{h_{0}} \tanh \left(\frac{M h}{h_{0}}\right)\right.\right.$

$$
\begin{aligned}
& +\frac{h}{h_{0}}\left[1-\tanh ^{2}\left(\frac{M h}{h_{0}}\right)\right]\left[\frac{M \alpha^{*}}{h_{0}}-\frac{M^{3}}{3} A\right] \\
& +\frac{\alpha^{* 2}}{h_{0}} \tanh \left(\frac{M h}{h_{0}}\right)+M\left[1-\tanh ^{2}\left(\frac{M h}{h_{0}}\right)\right] B \\
& +2 M^{2}(1+\lambda)\left\{\frac{h^{3}}{h_{0}^{3}} \tanh \left(\frac{M h}{h_{0}}\right)\right.
\end{aligned}
$$$$
+\frac{h^{3}}{h^{3}}\left[1-\tanh \left(\frac{M h}{h_{0}}\right)\right]\left[\frac{M \alpha^{*}}{h_{0}}-\frac{M^{3}}{3} A\right]
$$$$
+\tanh \left(\frac{M h}{h_{0}}\right) A+\frac{3 h^{2} \alpha^{*}}{h_{0}^{3}} \tanh \left(\frac{M h}{h_{0}}\right)+\frac{3 h^{2}}{h_{0}^{2}}\left[1-\tanh \left(\frac{M h}{h_{0}}\right)\right] M B
$$$$
\left.\left.+\left[\frac{3 h B}{h_{0}} \tanh \left(\frac{M h}{h_{0}}\right)\right]+\frac{3 h}{h_{0}}\left[1-\tanh ^{2}\left(\frac{M h}{h_{0}}\right)\right] M A\right\}-3 \lambda M B\right]
$$$$
12 M^{2}(1+\lambda)\left\{\tanh \left(\frac{M h}{h_{0}}\right)+\left[1-\tanh ^{2}\left(\frac{M h}{h_{0}}\right)\right]\left[\frac{M \alpha^{*}}{h_{0}}-\frac{M^{3}}{3} A\right]\right\}
$$

with

$$
A=\frac{\varepsilon^{*}}{h_{0}^{3}}+\frac{\alpha^{* 3}}{h_{0}^{3}}+3\left(\frac{\sigma^{*}}{h_{0}^{2}}\right)^{2} \frac{\alpha^{*}}{h_{0}} \text { and } B=\left(\frac{\alpha^{*}}{h_{0}}\right)^{2}+\left(\frac{\sigma^{*}}{h_{0}^{2}}\right)^{2}
$$

The relevant boundary conditions for the pressure field are

$$
\bar{p}=0 \quad \text { at } \quad x=0, L
$$

Introducing the non-dimensional scheme

$$
\begin{aligned}
& \bar{x}=\frac{x}{L}, \bar{h}=\frac{h}{h_{0}}, \bar{h}_{s}=\frac{h_{s}}{h_{0}}, P=\frac{\bar{p} h_{0}^{2}}{\mu U L}, \psi=\frac{k \delta}{h_{0}^{3}} \\
& \lambda=\frac{K}{\mu}, \quad \alpha=\frac{\alpha^{*}}{h_{0}}, \quad \sigma=\frac{\sigma^{*}}{h_{0}^{2}}, \quad \varepsilon=\frac{\varepsilon^{*}}{h_{0}^{3}}
\end{aligned}
$$


into the Eqs. (13) and (15) gives the non-dimensional averaged Reynolds type equation and boundary conditions in the form

$$
\begin{aligned}
& \frac{\partial}{\partial \bar{x}}\left\{\left[G(\bar{h}, M, \alpha, \sigma, \varepsilon)+\frac{\psi}{1+\lambda}\right] \frac{\partial P}{\partial \bar{x}}\right\}=\frac{1}{2} \frac{\partial \bar{h}}{\partial \bar{x}} \\
& P=0 \quad \text { at } \quad \bar{x}=0,1
\end{aligned}
$$

\section{BEARING CHARACTERISTICS}

The solution of Eq. (17) subject to boundary conditions (18) is obtained in the form

$$
P=I_{1}+D I_{2}
$$

where

$$
\begin{aligned}
& I_{1}=\int_{0}^{\bar{x}} \frac{\bar{h}}{2}\left[G(\bar{h}, M, \alpha, \sigma, \varepsilon)+\frac{\psi}{1+\lambda}\right]^{-1} d \bar{x}, \\
& I_{2}=\int_{0}^{\bar{x}}\left[G(\bar{h}, M, \alpha, \sigma, \varepsilon)+\frac{\psi}{1+\lambda}\right]^{-1} d \bar{x}, \\
& D=-\frac{I_{3}}{I 4}, \\
& I_{3}=\int_{0}^{1} \frac{\bar{h}}{2}\left[G(\bar{h}, M, \alpha, \sigma, \varepsilon)+\frac{\psi}{1+\lambda}\right]^{-1} d \bar{x} \text { and } \\
& I_{4}=\int_{0}^{1}\left[G(\bar{h}, M, \alpha, \sigma, \varepsilon)+\frac{\psi}{1+\lambda}\right]^{-1} d \bar{x} .
\end{aligned}
$$

The non-dimensional mean load carrying capacity is obtained in the form [22];

$$
\bar{W}=\int_{0}^{1} P d \bar{x}=-\int_{0}^{1} \bar{x} \frac{d P}{d \bar{x}} d \bar{x}
$$

Use of Eq. (19) in (21) gives

$$
\bar{W}=-\int_{0}^{1} \bar{x}\left\{\frac{\bar{h}}{2}\left[G(\bar{h}, M, \alpha, \sigma, \varepsilon)+\frac{\psi}{1+\lambda}\right]^{-1}\right.
$$

$$
\left.+D\left[G(\bar{h}, M, \alpha, \sigma, \varepsilon)+\frac{\psi}{1+\lambda}\right]^{-1}\right\} d \bar{x}
$$

The component of stress tensor required for computing frictional force is

$$
\tau_{y x}=\frac{1}{2}(2 \mu+K) \frac{\partial u}{\partial y}-K v
$$

The frictional force $\mathrm{F}$ per unit width on the sliding surface at $y=H$ is defined as

$$
F=\int_{0}^{L}\left(\tau_{y x}\right)_{y=H} d x
$$

where

$$
\left.\left(\tau_{y x}\right)_{y=H}=\left(\mu+\frac{K}{2}\right) \frac{\partial u}{\partial y}\right]_{y=H}
$$

since $\quad v=0$ at $\quad y=H$

Using the solution of Eq. (7) in Eq. (25) and then in Eq. (24) and the use of non-dimensional scheme gives the non-dimensional mean frictional force in the form

$$
\bar{F}=\int_{0}^{1}\left[\left(\frac{\bar{h}+\alpha}{2}\right) \frac{d P}{d \bar{x}}+\frac{M(1+\lambda)(2+\lambda)}{4 M \bar{h}(\bar{h}+\alpha)(1+\lambda)+\lambda g}\right] d \bar{x}
$$

where

$$
g=\left\{\tanh (M \bar{h})+\left[1-\tanh ^{2}(M \bar{h})\right]\left[M \alpha-\frac{M^{3}}{3}\left(\varepsilon+\alpha^{3}+\alpha^{2} \sigma\right)\right]\right\}
$$

The non-dimensional coefficient of friction is then obtained by

$$
\overline{C_{f}}=\frac{\bar{F}}{\bar{W}}
$$

\section{RESULTS AND DISCUSSION}

The Effect of surface roughness and micropolar 
lubricants on the performance characteristics of the porous inclined slider bearings is studied through the dimensionless parameters $\alpha, \varepsilon, \sigma, M, \psi$ and $\lambda$. As the roughness parameters tends to zero the results obtained in this paper reduce to the smooth case studied by Verma et al. [22]. For the numerical computations of the slider characteristics, the following set of values are used for various non-dimensional parameters $\alpha=-0.1-$ $0.1 ; \varepsilon=-0.1-0.1 ; \sigma=0.0-0.4 ; \lambda=0.0-0.4 ; M=4$, $6,8,10$; and $\psi=0.1,0.2$. The numerical values for the roughness parameters $\alpha, \varepsilon, \sigma$ are also so chosen that the corresponding film shapes are feasible.

\section{Pressure}

The effect of micropolar lubricants on the variation of the non-dimensional pressure $\mathrm{P}$ with $\bar{x}$ is depicted in Figure 2 for two values of the permeability

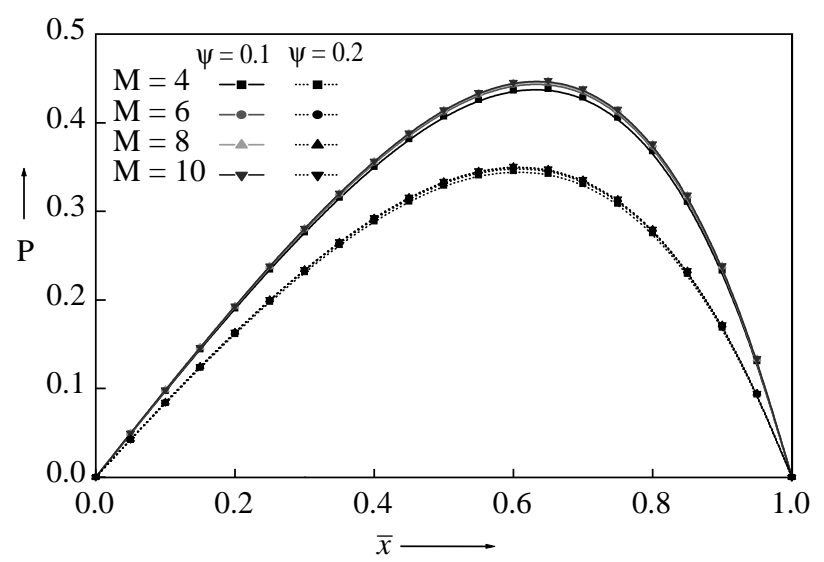

Fig. 2. Variation of non-dimensional pressure $P$ with $\bar{x}$ for different values of $M$ with $\alpha=-0.1, \sigma=0.1, \varepsilon=-0.1$ and $\lambda=2$.

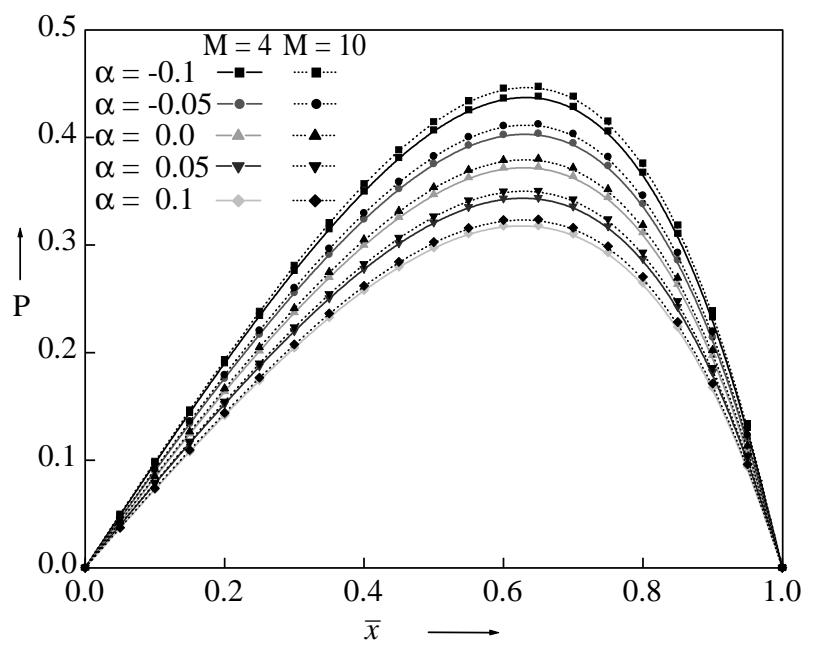

Fig. 3. Variation of non-dimensional pressure $P$ with $\bar{x}$ for different values of $\alpha$ with $\sigma=\mathbf{0 . 1}, \varepsilon=\mathbf{- 0 . 1}, \psi=0.1$ and $\lambda=2$. parameter $\psi$. The integrations $\mathrm{I}_{1}, \mathrm{I}_{2}$ and $\mathrm{I}_{3}$ involved in the calculation of non-dimensional pressure $\mathrm{P}$ given in Eq. (17) are calculated numerically [10]. It is observed that $P$ increases for increasing values of $M$ and for decreasing value of $\psi$. Further, it is observed that the point of maximum pressure is a function of the permeability parameter $\psi$.

The effect of roughness parameter $\alpha, \varepsilon$ and $\sigma$ on the variations of $P$ with $\bar{x}$ is shown in the Figures 3,4 and 5 respectively for two values of the parameter $M$. It is observed from Figures 3 and 4 that $P$ increases for negatively increasing values of $\alpha$ and $\varepsilon$ whereas $P$ decreases for positively increasing values of $\alpha$ and $\varepsilon$. The increasing values of $\sigma$ decreases the fluid film pressure $P$ (Figure 5).

\section{Load carrying capacity}

Figure 6 shows the variation of non-dimensional

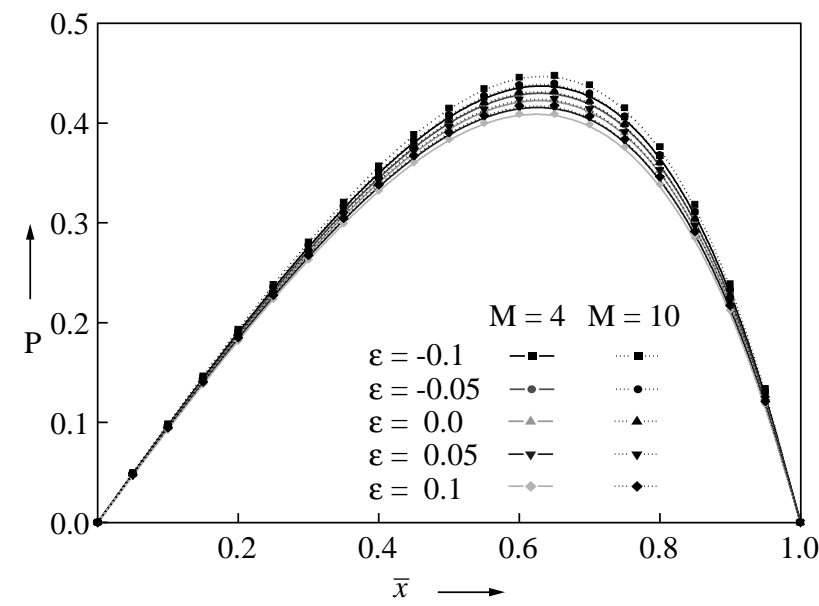

Fig. 4. Variation of non-dimensional pressure $P$ with $\bar{x}$ for different values of $\varepsilon$ with $\alpha=\mathbf{- 0 . 1}, \sigma=\mathbf{0 . 1}, \psi=0.1$ and $\lambda=2$.

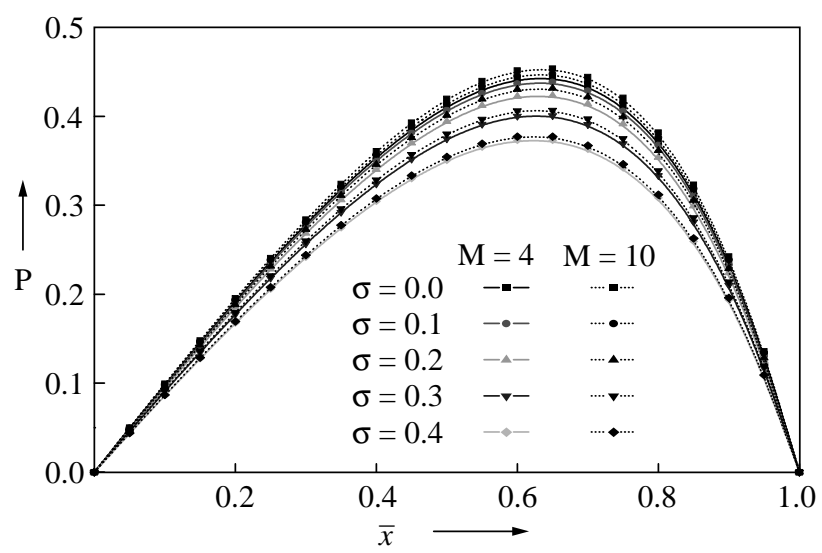

Fig. 5. Variation of non-dimensional pressure $P$ with $\bar{x}$ for different values of $\sigma$ with $\alpha=\mathbf{- 0 . 1}, \varepsilon=\mathbf{- 0 . 1}, \psi=0.1$ and $\lambda=2$. 
load carrying capacity $\bar{W}$ with $\lambda$ for different values of $M$. The numerical value of $\lambda=0$ corresponds to the Newtonian case. It is observed that $\bar{W}$ increases for increasing values of $\lambda$ and $M$. The effect of roughness parameter $\alpha, \varepsilon$ and $\sigma$ on the variation of $\bar{W}$ with $\lambda$ is depicted in the Figures 7, 8 and 9 respectively for two values of $M$. It is observed that, negatively skewed surface roughness increases $\bar{W}$ whereas positively skewed surface roughness decreases $\bar{W}$. Further, the significant increase in $\bar{W}$ is observed for larger values of $\lambda$ as compared to the Newtonian case $(\lambda=0)$.

\section{Frictional force}

Figure 10 shows the variation of non-dimensional frictional force $\bar{F}$ with $\lambda$ for different values of $\mathrm{M}$. It is observed that, for $\psi=0.1 \bar{F}$ decreases up to the value of $\lambda=0.5$ and then $\bar{F}$ increases for further increasing values of $\lambda$. However, $\bar{F}$ increases for all values of $\lambda$

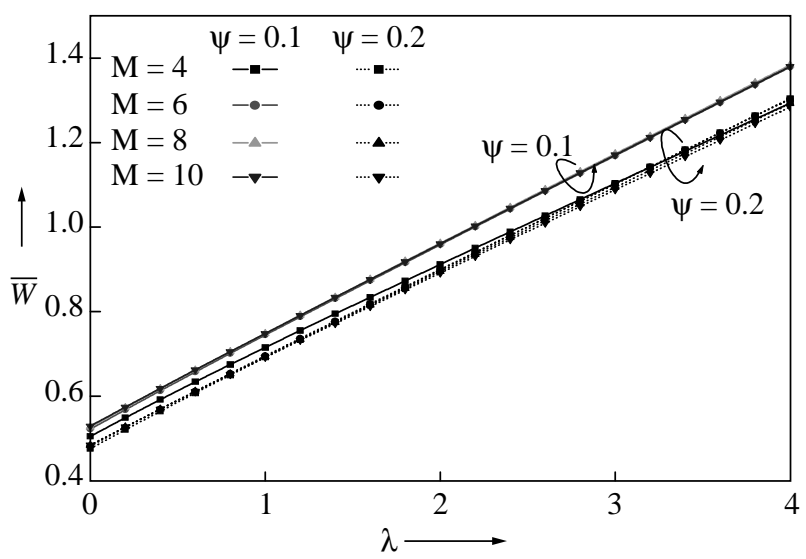

Fig. 6. Variation of non-dimensional load $\bar{W}$ with $\lambda$ for different values of $M$ with $\alpha=\mathbf{- 0 . 1}, \sigma=\mathbf{0 . 1}$ and $\varepsilon=\mathbf{- 0 . 1}$.

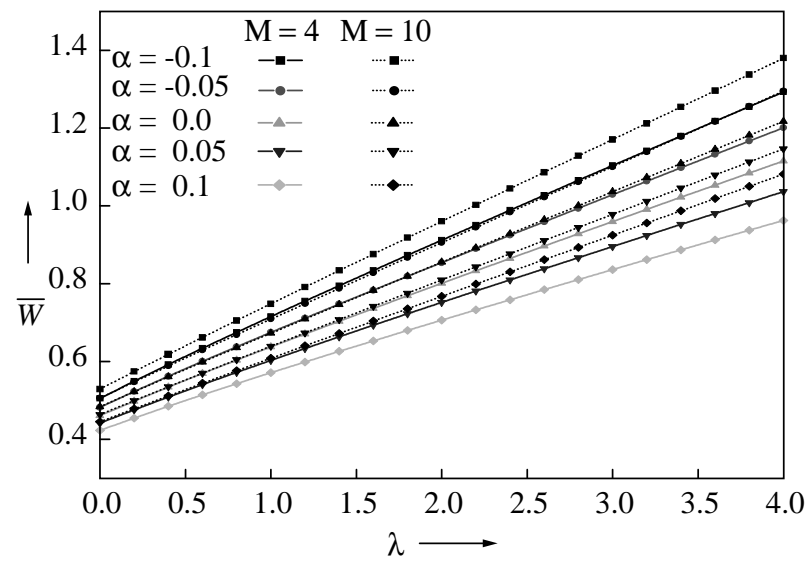

Fig. 7. Variation of non-dimensional load $\bar{W}$ with $\lambda$ for different values of $\alpha$ with $\sigma=0.1, \varepsilon=-\mathbf{0 . 1}$ and $\psi=0.1$. where $\psi=0.2$.

Figures 11,12 and 13 shows the variation of $\bar{F}$ with $\lambda$ for different values of $\alpha, \varepsilon$ and $\sigma$ respectively for two values of $M$. It is observed that negatively skewed surface roughness pattern decreases $\bar{F}$ whereas positively skewed surface roughness increases the frictional force on the sliding surface.

\section{Coefficient of friction}

The variation of non-dimensional coefficient of friction $\overline{C_{f}}$ with the parameter $\lambda$ for different values of $M$ is shown in the Figure 14 for two values of permeability parameter $\psi$. It is observed that $\overline{C_{f}}$ decreases rapidly for increasing values of $\lambda$ as compared to the Newtonian case $(\lambda=0)$. However, the marginal increase in $\overline{C_{f}}$ is observed for increasing values of $\mathrm{M}$.

The effect of roughness parameters $\alpha, \varepsilon$ and $\sigma$ on

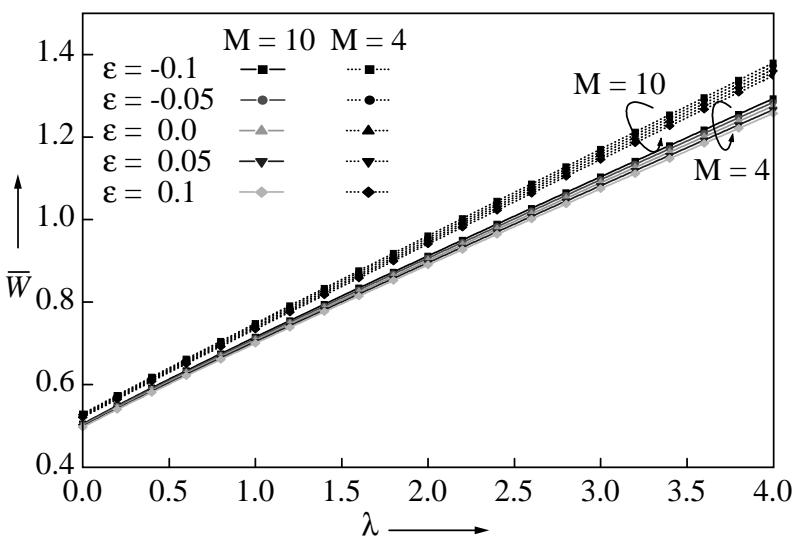

Fig. 8. Variation of non-dimensional load $\bar{W}$ with $\lambda$ for different values of $\varepsilon$ with $\alpha=\mathbf{- 0 . 1}, \sigma=\mathbf{0 . 1}$ and $\psi=0.1$.

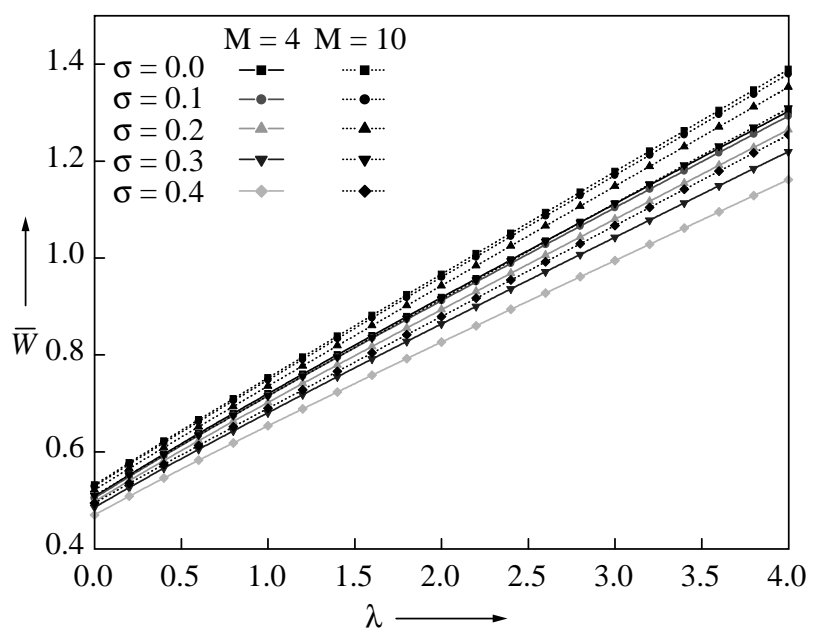

Fig. 9. Variation of non-dimensional load $\bar{W}$ with $\lambda$ for different values of $\sigma$ with $\alpha=\mathbf{- 0 . 1}, \varepsilon=\mathbf{- 0 . 1}$ and $\psi=\mathbf{0 . 1}$. 


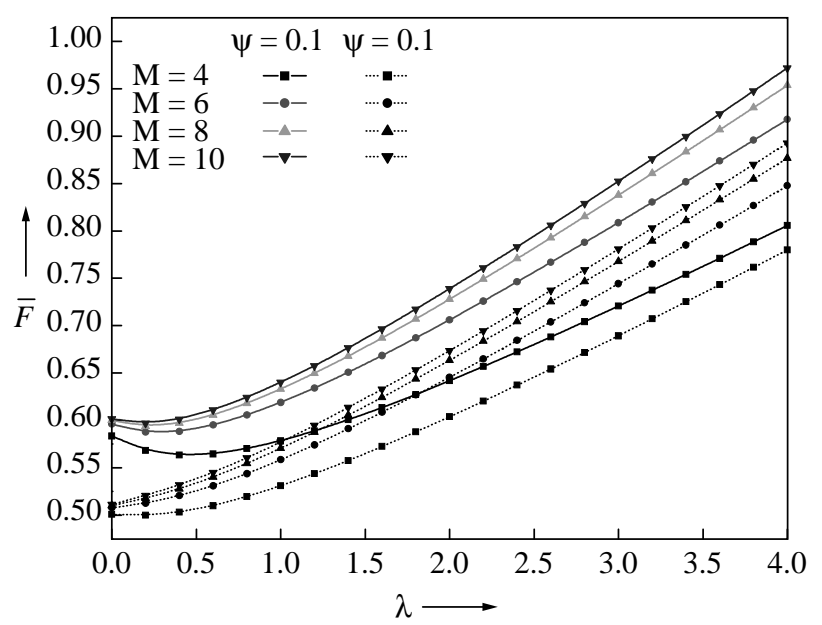

Fig. 10. Variation of non-dimensional frictional force $\bar{F}$ with $\lambda$ for different values of $M$ with $\alpha=\mathbf{- 0 . 1}, \sigma=0.1$ and $\varepsilon=\mathbf{- 0 . 1}$.

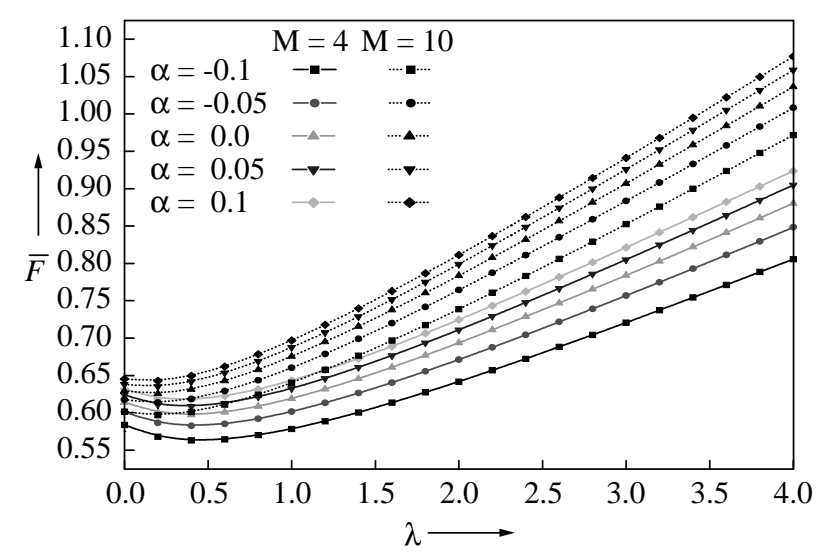

Fig. 11. Variation of non-dimensional frictional force $\overline{\boldsymbol{F}}$ with $\lambda$ for different values of $\alpha$ with $\sigma=0.1, \varepsilon=-\mathbf{0 . 1}$ and $\psi=0.1$.

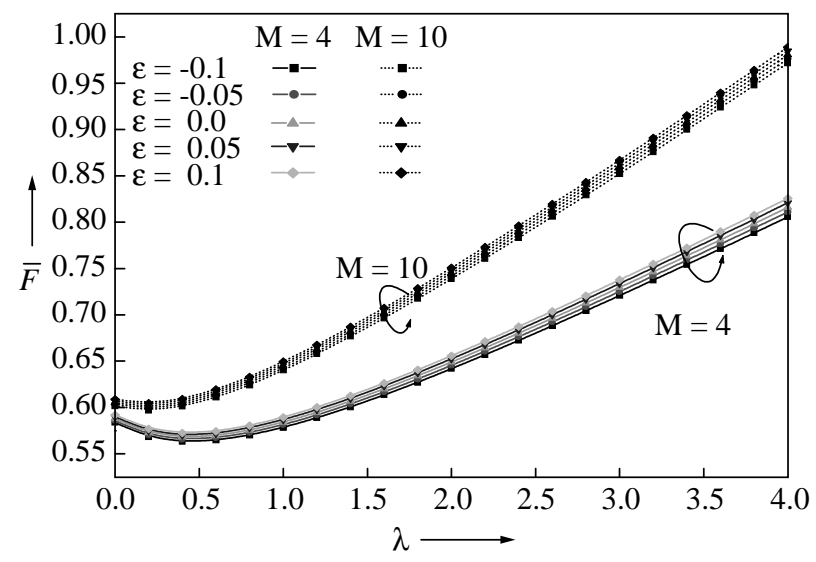

Fig. 12. Variation of non-dimensional frictional force $\overline{\boldsymbol{F}}$ with $\lambda$ for different values of $\varepsilon$ with $\alpha=\mathbf{- 0 . 1}, \sigma=0.1$ and $\psi=0.1$.

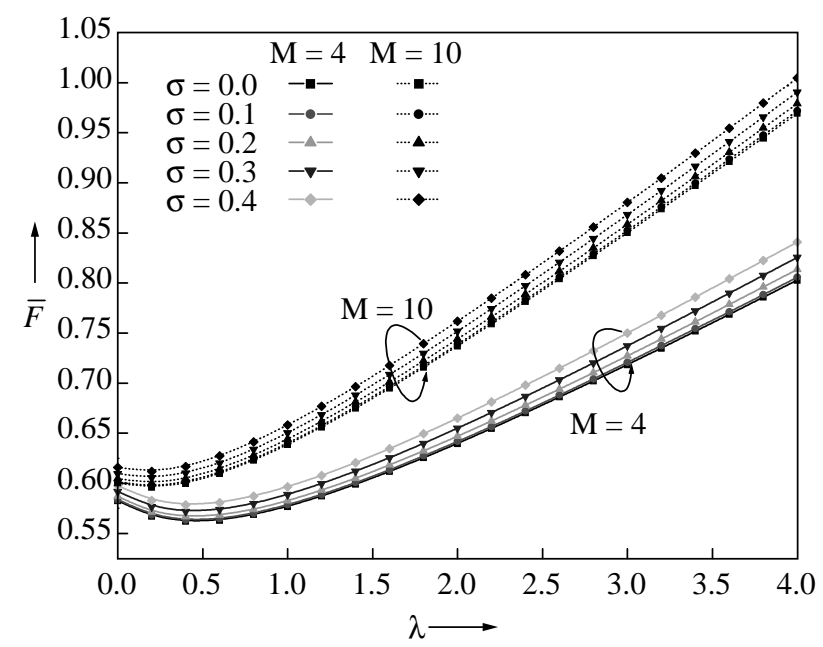

Fig. 13. Variation of non-dimensional frictional force $\bar{F}$ with $\lambda$ for different values of $\sigma$ with $\alpha=\mathbf{- 0 . 1}, \varepsilon=\mathbf{- 0 . 1}$ and $\psi=\mathbf{0 . 1}$.

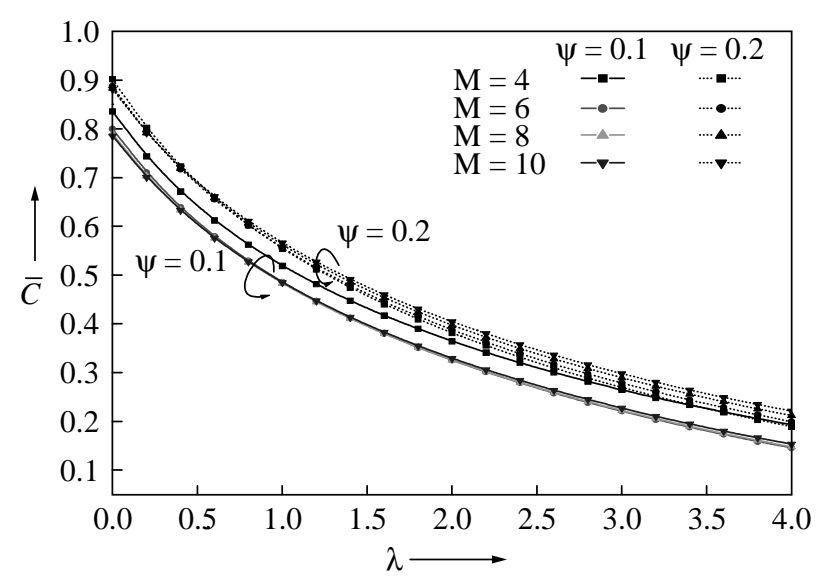

Fig. 14. Variation of non-dimensional Coefficient of friction $\overline{C_{f}}$ with $\lambda$ for different values of $\mathrm{M}$ with $\alpha=\mathbf{- 0 . 1}, \sigma=\mathbf{0 . 1}$ and $\varepsilon=\mathbf{- 0 . 1}$

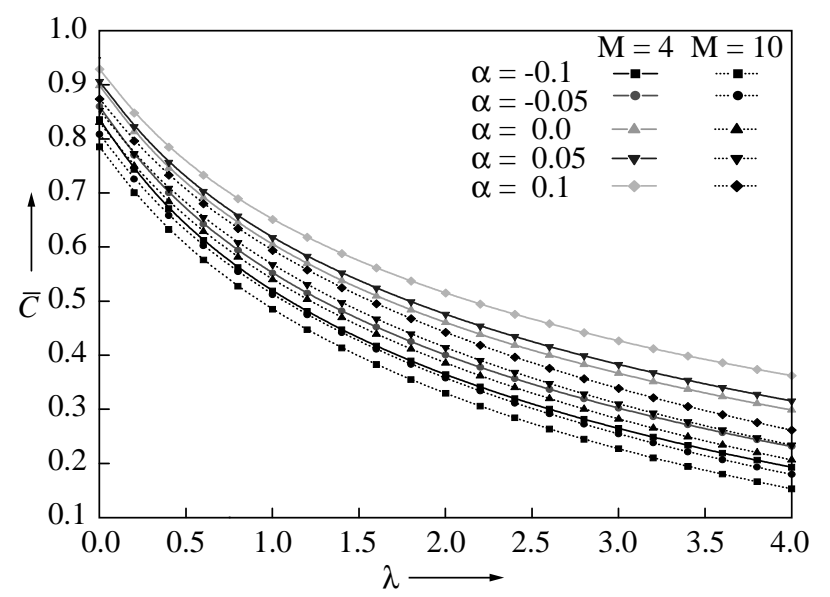

Fig. 15. Variation of non-dimensional Coefficient of friction $\overline{C_{f}}$ with $\lambda$ for different values of $\alpha$ with $\sigma=0.1, \varepsilon=-0.1$ and $\psi=0.1$. 


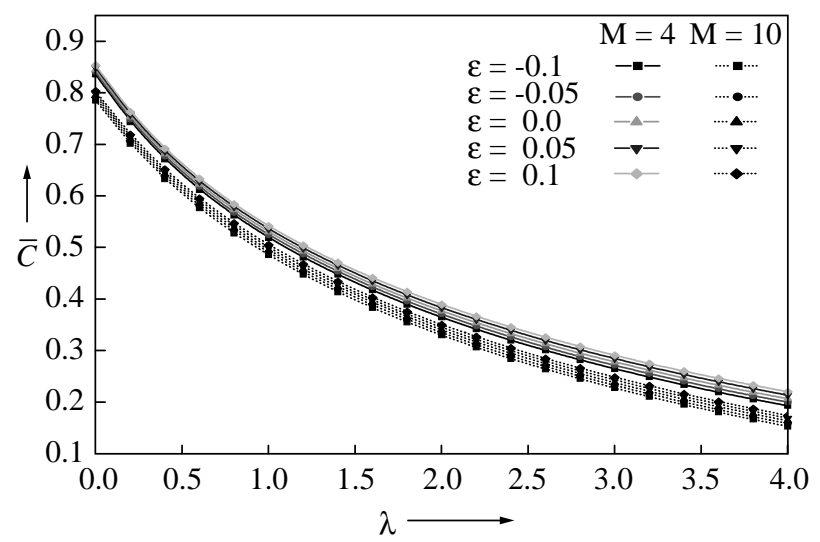

Fig. 16. Variation of non-dimensional Coefficient of friction $\overline{C_{f}}$ with $\lambda$ for different values of $\varepsilon$ with $\alpha=\mathbf{- 0 . 1}, \sigma=0.1$ and $\psi=0.1$.

the variation of $\overline{C_{f}}$ with $\lambda$ is depicted in Figures 15, 16 and 17. It is observed that $\overline{C_{f}}$ decreases for negatively skewed surface roughness pattern whereas it increases for positively skewed surfaces roughness pattern. Further, the increasing values of $\sigma$ increases the coefficient of friction $\overline{C_{f}}$ marginally. These increase/decrease in $\overline{C_{f}}$ are more pronounced for larger values of $\lambda$.

\section{CONCLUSIONS}

The performance characteristics of the rough porous inclined slider bearings lubricated with micropolar fluids is analysed on the basis of Eringen [8] constitutive equations for micropolar fluids and the stochastic random variable to represent the surface roughness of the bearing with non-zero mean variance and skewness. On the basis of the numerical computations performed, the following conclusions can be drawn.

1. The significant increase in the load carrying capacity and decrease in the coefficient of friction can be attained by the use of micropolar lubricants.

2. The porous facing on the slider bearing affects its performance.

3 . The presence of negatively skewed surface roughness pattern on the bearing surface improves its performance.

4. The positively skewed surface roughness pattern on the porous slider bearing affects its performance.

5. The above effects are more accentuated for larger values of the parameter $\lambda$.

\section{ACKNOWLEDGEMENTS}

The authors are grateful to the referees for their valuable comments on the earlier draft of the paper. One of the authors (TVB) is thankful to Poojya Dr.

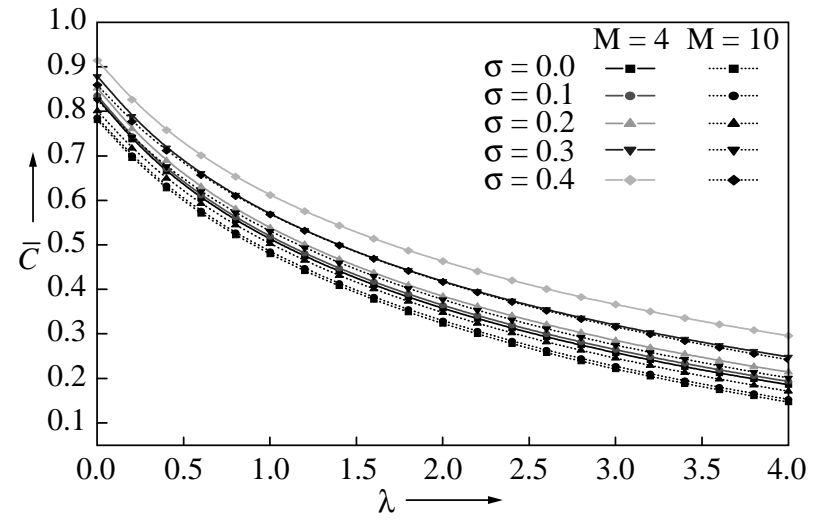

Fig. 17. Variation of non-dimensional Coefficient of friction $\overline{C_{f}}$ with $\lambda$ for different values of $\sigma$ with $\alpha=\mathbf{- 0 . 1}, \varepsilon=\mathbf{- 0 . 1}$ and $\psi=0.1$.

Sharnbasawappa Appa, President, Sharnbasveshwar Vidya Vardhak Sangha, Gulbarga for his encouragements. This work is supported by the UGC New Delhi under the Project No.F-31-84/2005(SR).

\section{NOMENCLATURE}

C maximum asperity height from the mean level

$\overline{C_{f}} \quad$ non-dimensional frictional coefficient, $\overline{C_{f}}=\bar{F} / \bar{W}$

E expectancy operator defined by equation (5)

$F \quad$ frictional force

$\bar{F} \quad$ non-dimensional frictional force, $\bar{F}=\frac{F h_{o}}{\mu U L}$

$H \quad$ fluid film thickness, $H=h+h_{s}$

$h \quad$ mean film thickness, $h(x)=h_{1}-\left(\frac{h_{1}-h_{0}}{L}\right) x$

$\bar{h} \quad$ non-dimensional mean film thickness, $\bar{h}=h / h_{o}$

$\bar{H} \quad$ non-dimensional fluid film thickness, $\bar{H}=H / h_{o}$

$\mathrm{h}_{\mathrm{o}} \quad$ outlet film thickness

$h_{1} \quad$ inlet film thickness

$h_{s} \quad$ random variable

$K \quad$ viscosity coefficient for micropolar fluid.

$k$ permeability of the porous matrix.

$L \quad$ bearing length

M non-dimensional micropolar parameter,

$M=m h_{o} / 2$ 


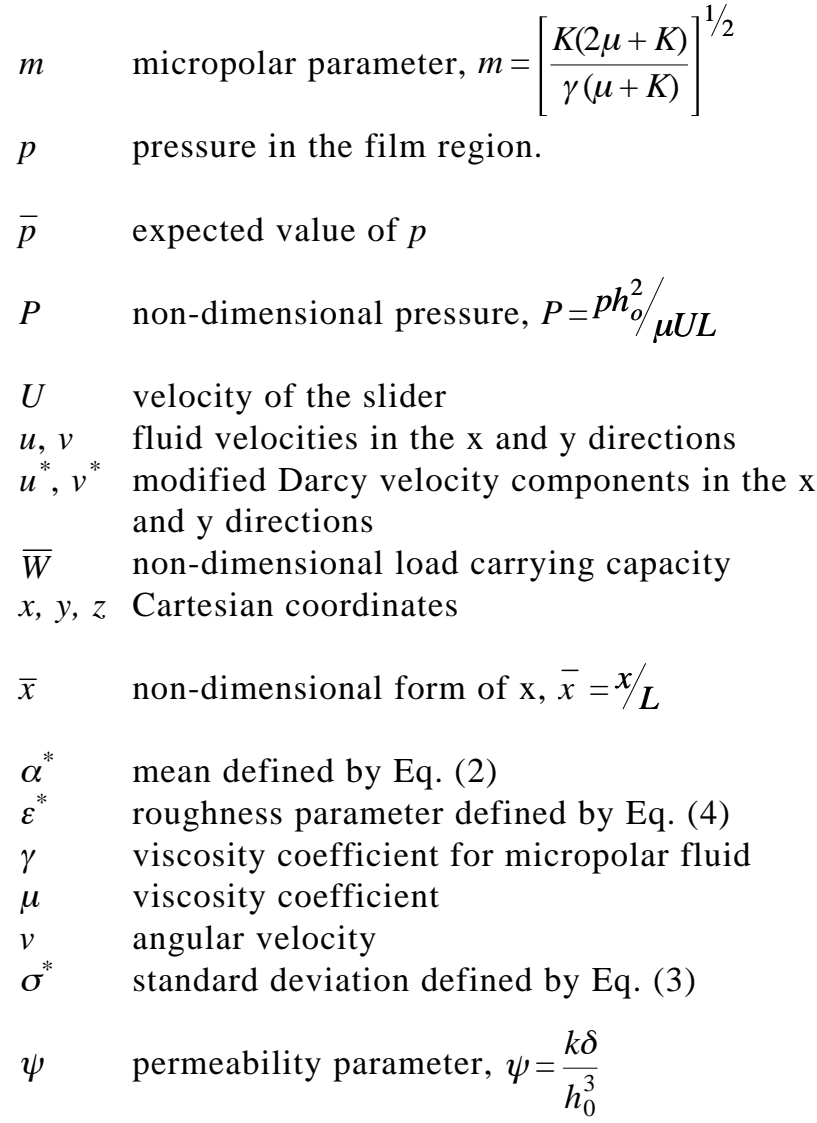

\section{REFERENCES}

1. Allen, S.J. and Kline, K.A., "Lubrication Theory for Micropolar Fluids," Journal of Applied Mechanics, Vol. 38, pp. 646-650 (1971).

2. Andharia, P.I., Gupta, J.L., and Deheri, G.M., "Effect of Surface Roughness on Hydrodynamic Lubrication of Slider Bearings," Tribology Transaction, Vol. 44, No. 2, pp. 291-297 (2001).

3. Christensen, H., "Stochastic Models for Hydrodynamic Lubrication of Rough Surfaces," International Journal of Mechanical Engineering, Vol. 184, pp. 1013-1026 (1970).

4. Christensen, H. and Tonder, K., "Tribology of Rough Surfaces; Stochastic Models of Hydrodynamic Lubrication," SINTEF Report, No. 10, pp. 69-18 (1969).

5. Christensen, H. and Tonder, K., "The Hydrodynamic Lubrication of Rough Bearing Surfaces of Finite Width," ASME Journal of Lubrication Technolog, Vol. 93, pp. 324-330 (1971).

6. Christensen, H. and Tonder, K., "Waviness and Roughness in Hydrodynamic Lubrication," Proceedings of Institution of Mechanical Engineers, Vol. 186, No. 72, pp. 807-812 (1972).

7. Eringen, A.C., "Simple Microfluids," International Journal of Engineering Science, Vol. 2, pp. 205-217 (1964).
8. Eringen, A.C., "Theory of Micropolar Fluids," Journal of Mathematics and Mechanics, Vol. 16, No. 1, pp. 1-18 (1966).

9. Gururajan, K. and Prakash, J., "Effect of Velocity Slip in a Narrow Rough Porous Journal Bearing," Journal of Engineering Tribology, Vol. 217, pp. 59-69 (2003).

10. Jain, M.K., Iyengar, S.R.K., and Jain, R.K., Numerical Methods for Scientific and Engineering Computations, New Age International Publishers, New Delhi, ND (2002).

11. Letalleur, N., Plourbouc, F., and Prat, M., "Average Flow Model of Rough Surface Lubrication," ASME Journal of Tribology, Vol. 124, pp. 539-545 (2002).

12. Lin, J.R., Hsu, C.H., and Lai, C., "Surface Roughness Effects on the Oscillating Squeeze Film Behavior of Long Partial Journal Bearings," Computers \& Structures, Vol. 80, pp. 297-303 (2002).

13. Lin, T.R., "Hydrodynamic Lubrication of Journal Bearings Including Micropolar Lubricants and Three-Dimensional Irregularities," WEAR, Vol. 192, pp. 21-28 (1996).

14. Naduvinamani, N.B. and Gurubasavaraj, G., "Surface Roughness Effects on Squeeze Films in Curved Circular Plates," Industrial Lubrication and Tribology, Vol. 56, No. 6, pp. 346-352 (2004).

15. Patir, N. and Cheng, H., "An Average Flow Model for Determining Effects of Three-Dimensional Roughness on Partial Hydrodynamic Lubrication," ASME Journal of Lubrication Technolog, Vol. 100, pp. 12-17 (1978).

16. Patir, N. and Cheng, H., "Application of Average Flow Model to Lubrication Between Rough Sliding Surfaces," ASME Journal of Lubrication Technolog, Vol. 101, pp. 220-230 (1979).

17. Prakash, J. and Sinha, P., "Lubrication Theory of Micropolar Fluids and Its Application to a Journal Bearing," International Journal of Engineering Science, Vol. 13, pp. 217-232 (1975).

18. Prakash, J., Tonder, K., and Christensen, H., "Micropolarity Roughness Interaction in Hydrodynamic Lubrication," Transaction of ASME, Vol. 102, pp. 368-372 (1980).

19. Ramanaiah, G. and Dubey, J.N., "Optimum Slider Profile of a Slider Bearing Lubricated with a Micropolar Fluid," WEAR, Vol. 42, pp. 1-7 (1977).

20. Shukla, J.B. and Isa, M., "Generalized Reynolds Type Equation for Micropolar Lubricants and Its Application to Optimum One-Dimensional Slider Bearing Effects of Solid Particle Additives in Solution," Journal of Mechanical Engineering Science, Vol. 17, pp. 280-284 (1975).

21. Tzen, S.T. and Saibel, E., "Surface Roughness Effects on Slider Bearing Lubrication," ASLE Transactions, Vol. 10, pp. 334-338 (1967).

22. Verma, P.D.S., Agrawal, V.K., and Bhatt, S.B., "Porous Inclined Slider Bearing Lubricated with Micropolar Fluid," WEAR, Vol. 53, pp. 101-106 (1979). 